\title{
p66Shc deletion or deficiency protects from obesity but not metabolic dysfunction in mice and humans
}

\author{
Stefano Ciciliot ${ }^{1,2} \cdot$ Mattia Albiero $^{1,2} \cdot$ Lisa Menegazzo $^{1,2} \cdot$ Nicol Poncina $^{1,2}$. \\ Valentina Scattolini ${ }^{1,2}$ • Andrea Danesi ${ }^{2} \cdot$ Elisa Pagnin $^{1}$ • Manuela Marabita ${ }^{2}$. \\ Bert Blaauw $^{2,3}$ • Marco Giorgio ${ }^{4}$. Mirella Trinei ${ }^{4} \cdot$ Mirto Foletto $^{5}$ • Luca Prevedello ${ }^{5}$. \\ Donato Nitti $^{5}$ - Angelo Avogaro ${ }^{1,2}$ - Gian Paolo Fadini ${ }^{1,2}$
}

Received: 19 February 2015 / Accepted: 26 May 2015 /Published online: 30 June 2015

(C) Springer-Verlag Berlin Heidelberg 2015

\begin{abstract}
Aims/hypothesis Oxygen radicals generated by p66Shc drive adipogenesis, but contradictory data exist on the role of p66Shc in the development of obesity and the metabolic syndrome. We herein explored the relationships among p66Shc, adipose tissue remodelling and glucose metabolism using mouse models and human adipose tissue samples.

Methods In wild-type (WT), leptin-deficient (ob/ob), $p 66 \mathrm{Shc}^{-/-}$and $p 66 \mathrm{Sh}^{-/-}$ob/ob mice up to 30 weeks of age, we analysed body weight, subcutaneous and visceral adipose tissue histopathology, glucose tolerance and insulin sensitivity, and liver and muscle fat accumulation. A group of mice on a high fat diet (HFD) was also analysed. A parallel study was conducted on adipose tissue collected from patients undergoing elective surgery.

Results We found that $p 66 \mathrm{Shc}^{-1-}$ mice were slightly leaner than WT mice, and $p 66 \mathrm{Sh}^{-/-}$ob/ob mice became less obese than $o b / o b$ mice. Despite their lower body weight, $p 66 \mathrm{Shc}^{-1-}$
\end{abstract}

Stefano Ciciliot and Mattia Albiero contributed equally to this work.

Electronic supplementary material The online version of this article (doi:10.1007/s00125-015-3667-8) contains peer-reviewed but unedited supplementary material, which is available to authorised users.

Gian Paolo Fadini

Gianpaolo.fadini@unipd.it

1 Department of Medicine, University of Padua, Via Giustiniani, 2, 35128 Padua, Italy

2 Venetian Institute of Molecular Medicine, Padua, Italy

3 Department of Biomedical Sciences, University of Padua, Padua, Italy

4 European Institute of Oncology, IFOM-IEO Campus, Milan, Italy

5 Department of Surgical, Oncologic Gastroenterologic Sciences, University of Padua, Padua, Italy mice accumulated ectopic fat in the liver and muscles, and were glucose intolerant and insulin resistant. Features of adverse adipose tissue remodelling induced by obesity, including adipocyte enlargement, apoptosis, inflammation and perfusion were modestly and transiently improved by $p 66 \mathrm{Shc}$ (also known as $S h c 1$ ) deletion. After 12 weeks of the HFD, p66Shc ${ }^{-1-}$ mice were leaner than but equally glucose intolerant and insulin resistant compared with WT mice. In 77 patients, we found a direct correlation between BMI and p66Shc protein levels. Patients with low p66Shc levels were less obese, but were not protected from other metabolic syndrome features (diabetes, dyslipidaemia and hypertension).

Conclusions/interpretation In mice and humans, reduced p66Shc levels protect from obesity, but not from ectopic fat accumulation, glucose intolerance and insulin resistance.

Keywords Diabesity $\cdot$ Inflammation $\cdot$ Longevity $\cdot$ Oxidative stress

$\begin{array}{ll}\text { Abbreviations } \\ \text { Akt } & \text { Protein kinase B } \\ \text { ANOVA } & \text { Analysis of variance } \\ \text { ATP-III } & \text { Adult Treatment Panel III } \\ \text { BMI } & \text { Body mass index } \\ \text { CLS } & \text { Crown-like structures } \\ \text { CSA } & \text { Cross-sectional area } \\ \text { ERK } & \text { Extracellular signal-regulated kinase } \\ \text { HFD } & \text { High fat diet } \\ \text { ipGTT } & \text { i.p. glucose tolerance test } \\ \text { ITT } & \text { Insulin tolerance test } \\ \text { mTOR } & \text { Mammalian target of rapamycin } \\ \text { p66Shc } & \text { SHC (Src homology 2 domain containing) } \\ & \text { transforming protein 1, 66 kDa isoform } \\ \text { ROS } & \text { Reactive oxygen species }\end{array}$




$\begin{array}{ll}\text { S6K } & \text { S6 kinase } \\ \text { SAT } & \text { Subcutaneous adipose tissue } \\ \text { VAT } & \text { Visceral adipose tissue } \\ \text { WT } & \text { Wild-type }\end{array}$

\section{Introduction}

The $66 \mathrm{kDa}$ p66Shc is a unique protein isoform of SHC (Src homology 2 domain containing) transforming protein 1 , encoded by the mammalian $S h c A$ gene (also known as $S h c 1$ ), and acts as a receptor tyrosine kinase adaptor and redox enzyme [1]. Tyrosine phosphorylation of p66Shc mediates downstream effects of growth factors, whereas serine phosphorylation causes p66Shc to translocate to the mitochondrial intermembrane space, where it interacts with cytochrome $\mathrm{C}$ and produces $\mathrm{H}_{2} \mathrm{O}_{2}$ [2]. Genetic deletion or knockdown of p66Shc results in decreased levels of reactive oxygen species (ROS). Thus, in accordance with the free-radical theory of ageing $\mathrm{p} 66 \mathrm{Shc} \mathrm{c}^{-/-}$ mice live longer than wild-type (WT) mice in a controlled environment [3]. $\mathrm{p} 66 \mathrm{Shc}^{-/-}$mice are also protected from typical oxidative stress related diseases, including diabetic complications [4-7]. ROS generated via p66Shc are required for adipogenesis, such that $\mathrm{p} 66 \mathrm{Sh} \mathrm{c}^{-/-}$mice gain less weight when fed a high fat diet (HFD), with concomitant adipose tissue browning and a mildly raised body temperature [8]. Impaired adipogenesis and thermo-insulation probably make $p 66 \mathrm{Shc}^{-1-}$ mice less resistant to cold and wild environments [9]. Based on these observations, $p 66$ Shc can be considered a 'thrifty gene', which is evolutionarily conserved in mammals [10].

Previous reports show that 20 -week-old $p 66 \mathrm{Shc}^{-1-}$ mice rendered obese by crossing with leptin-deficient $\left(\mathrm{Lep}^{\mathrm{ob} / \mathrm{ob}}\right)$ mice, are more glucose tolerant and insulin sensitive than their p66Shc ${ }^{+/+}$counterparts [11]. However, two independent p66Shc ${ }^{-1-}$ strains respond differently to HFD: while the original ShcP strain is protected from fat accumulation [8], the ShcL strain has a fat phenotype [12].

To date, no study has compared adipose tissue histopathology during the development of obesity in WT and $\mathrm{p} 66 \mathrm{Sh} \mathrm{c}^{-/-}$ mice. We herein analysed subcutaneous adipose tissue (SAT) and visceral adipose tissue (VAT) remodelling in parallel with changes in body weight and metabolic features in WT and p66Shc $^{-/-}$mice on Lep ${ }^{W T / W T}$ and $L e p^{o b / o b} \mathrm{C} 57 \mathrm{Bl} / 6 \mathrm{~J}$ backgrounds. These data, together with a parallel study carried out in humans, suggest that $p 66 S h c$ deletion protects from the development of obesity, but not from glucose intolerance and insulin resistance.

\section{Methods}

Animal experiments All procedures involving animals were approved by the local ethics committee and the Italian
Ministry of Health. Experiments were conducted according to the National Institutes of Health Principles of Laboratory Animal Care. Animals were maintained under standard laboratory conditions, kept in $12 \mathrm{~h}$ light-dark cycles with ad libitum access to food and water. The $p 66$ Shc knockout mice were generated by M. Giorgio [3] and correspond to the ShcP strain [12]. $p 66 S h c^{-1-}$ and $p 66 S h c^{-1-} L e p^{o b / o b}$ animals were bred at the animal facility of the University of Padua. Lep ${ }^{o b /}$ ${ }^{o b}$ mice were obtained from The Jackson Laboratory (Bar Harbor, ME, USA). Age- and sex-matched WT animals were obtained from the in-house colony of the animal facility of the Venetian Institute of Molecular Medicine (Padua, Italy). In a separate set of experiments, WT and $p 66 S h c^{-/-}$mice were fed an HFD with $60 \%$ of the total energy derived from fat (ssniff EF acc. D12492 (I) mod., Spezialdiäten, Soest, Germany) from 4 to 16 weeks of age (12 week HFD duration). Both male and female mice were used. WT and $p 66 S h c^{-1-}$ were randomly assigned to the HFD group or standard diet group.

GTTs and insulin tolerance tests were performed in fasted animals by measuring blood glucose at $0,30,60$ and $120 \mathrm{~min}$ after injection of $1 \mathrm{~g} / \mathrm{kg}$ D-glucose, or $0.75 \mathrm{U} / \mathrm{kg}$ human recombinant insulin (Humulin R; Lilly, Indianapolis, IN, USA), respectively. Glucose uptake into isolated mouse muscles was assayed by the uptake of 2-deoxy-D-glucose, using a colorimetric kit. Serum adipokines were measured using a commercially available multiple suspension assay. For full details on these procedures, please see the electronic supplementary material (ESM) Methods.

HOMA-IR and QUICKI values were calculated as follows:

$$
\begin{aligned}
& \text { HOMA-IR }=\frac{\left(\operatorname{glucose}\left(\frac{\mathrm{mmol}}{l}\right) \times \operatorname{insulin}\left(\frac{\mathrm{pmol}}{l}\right)\right)}{22.5} \\
& \text { QUICKI }=\frac{1}{\log \left(\frac{\text { fasting insulin }\left(\frac{\mathrm{pmol}}{l}\right)}{6.945}\right)+\log \left(\operatorname{glucose}\left(\frac{\mathrm{mmol}}{l}\right) \times 18\right)}
\end{aligned}
$$

Investigators were blinded when analysing mouse samples and when assessing the outcome of experiments. Exclusion criteria were pre-determined: animals were excluded from analysis in case of death, cannibalism, or sickness.

Adipose tissue histopathology Histopatology experiments were performed on at least three biological replicates, and at least five random fields per sample were analysed.

Ten micrometre thick sections of paraffin-embedded mouse VAT and SAT were cut with a Leica microtome. Sections were deparaffinised in xylene, rehydrated in serial dilutions of ethanol and, finally, rinsed with tap water. An antigen retrieval step was applied for F4/80 and TUNEL staining, by incubating sections for $10 \mathrm{~min}$ with $10 \mu \mathrm{mol} / 1$ proteinase $\mathrm{K}$ diluted in $0.1 \mathrm{~mol} / 1$ Tris-HCl. TUNEL staining was then performed following manufacturer's protocol (ApopTag; 
Millipore, Temecula CA, USA). For F4/80 antibody staining (Abcam, Cambridge, UK), sections were saturated with $1 \%$ BSA and $10 \%$ donkey serum in PBS for $1 \mathrm{~h}$ at room temperature, and then incubated in a 1:150 dilution of primary antibody at $4^{\circ} \mathrm{C}$ overnight. Sections were then washed three times with PBS and endogenous peroxidase activity was quenched by immersing sections for $30 \min$ in $0.3 \% \mathrm{H}_{2} \mathrm{O}_{2}$ in ethanol, and then washing twice with PBS. Secondary donkey anti-rat HRP-conjugated antibody was used (Jackson ImmuoResearch Europe, Suffolk, UK). Samples were then washed and incubated with 3,3'-diaminobenzidine substrate (ImmPACT DAB; Vector Laboratories, Peterborough, UK) following the manufacturer's instructions. Haematoxylin and eosin staining was performed using a Rapid Frozen Section Kit (Bio-Optica, Milan, Italy), following the manufacturer's instructions. Picrosirius red staining was performed by immersing sections in Direct Red 80 (Sigma-Aldrich, Milan, Italy) dissolved in a saturated aqueous solution of picric acid (Sigma-Aldrich) for $1 \mathrm{~h}$ at room temperature, and then washing in two changes of acidified water $(0.5 \% \mathrm{v} / \mathrm{v}$ glacial acetic acid in distilled water). Samples were then dehydrated in three changes of absolute ethanol, cleared in two changes of xylene and mounted with mounting medium (Bio-Optica). Histological images were taken with a Leica DM5000B microscope equipped with a DFC300 FX charge-coupled device camera. Morphometric analysis of adipocyte size was performed using a semiautomated procedure with Fiji/ImageJ (1.48r, National Institutes of Health, Bethesda MD, USA). For full details, please see the ESM Methods. Triacylglycerol content was determined in lysed liver and muscle samples using an automated spectrophotometer. Oil Red O staining of tissue sections was also performed. Adipose tissue haemoglobin content was measured using a spectrophotometer and normalised to protein content. A custom multiplex suspension phospho-protein array was used to assess signalling pathways, determined the ratio of phosphorylated vs total protein kinase B (Akt) $\left(\mathrm{Ser}^{473}\right)$, extracellular signal-regulated kinases 1 and 2 (ERK1/2) $\left(\mathrm{Thr}^{202} / \mathrm{Tyr}^{204}\right.$, $\mathrm{Thr}^{185} / \mathrm{Tyr}^{187}$ ), mammalian target of rapamycin (mTor; $\mathrm{Ser}^{2448}$ ) and S6 kinase $\left(\mathrm{S} 6 \mathrm{~K} ; \mathrm{Thr}^{389}\right)$. For full details on these procedures, please see the ESM Methods.

Clinical study The protocol was approved by local ethics institutions and conducted in accordance with the Declaration of Helsinki. Patients undergoing elective surgery for morbid obesity or cholecystectomy were recruited at the 1st General Surgery Unit of the University Hospital of Padua. All patients provided informed consent for the collection of adipose tissue samples during surgery. We recorded the following clinical data: age, sex, baseline BMI, prevalence of diabetes (diagnosed according to ADA criteria), dyslipidaemia (defined in this population as total cholesterol $>5.18 \mathrm{mmol} / \mathrm{l}$, triacylglycerol $>1.69 \mathrm{mmol} / \mathrm{l}$ ), hypertension (defined as systolic BP $>140 \mathrm{mmHg}$ or diastolic BP $>90 \mathrm{mmHg}$ ), the metabolic syndrome (defined according to revised Adult Treatment Panel III [ATP-III] criteria). Prevalence of cardiovascular disease was defined as a history of myocardial infarction, angina, stroke or peripheral arterial disease, or evidence of significant stenosis in the coronary, carotid or leg arteries.

RNA isolation and qPCR analysis Total RNA was extracted from fat samples using an RNeasy kit (Qiagen, Venlo, the Netherlands) following the manufacturer's instructions. Total RNA was reverse transcribed using Superscript II reverse transcriptase (Thermo Scientific, Monza, Italy) and the cDNA was analysed by quantitative real-time PCR (qPCR) using primers specific for $p 665 h c$ and TaqMan chemistry (Universal probe library no. 8; Roche Diagnostics, Milan, Italy) and the primers FW, 5'-CTGGAGGAAGGGGCTTCT-3'; RV, 5'-AGGCAG AGGAGGCAGGAT-3'. Results were expressed as fold change relative to the controls after normalisation using $18 \mathrm{~S}$ rRNA (Life Technologies, Monza, Italy) gene expression levels.

Western blotting Tissue extracts were prepared by homogenisation and lysis with extraction buffer, protease inhibitor (Roche Diagnostics), and phosphatase inhibitor (SigmaAldrich). The protein concentration was determined using standard Bradford reagent (Sigma-Aldrich). Proteins were separated by $10 \%$ SDS-PAGE and transferred onto a nitrocellulose membrane. This was incubated in PBS containing $0.1 \%$ Tween- 20 and $5 \%$ non-fat dry milk with the primary anti-Shc polyclonal antibody (BD Transduction Laboratories, Milan, Italy) for $3 \mathrm{~h}$ at room temperature. Membranes were then incubated with HRP-conjugated secondary antibody (Cell Signaling, Leiden, the Netherlands). Membranes were reprobed with an anti- $\beta$ actin antibody to control for protein loading (SigmaAldrich). Immunoreactive proteins were visualised with a chemiluminescence substrate (Pierce, Rockford, USA) and captured on x-ray film.

Statistical analysis Data are reported as the mean \pm SE for continuous variables or as a percentage for categorical variables. Normal distribution of the variables of interest was checked using the Kolmogorov-Smirnov test. Non-normal continuous variables were log-transformed before analysis. Comparison of data between two or more groups was performed using a two-tailed unpaired Student's $t$ test or twoway ANOVA (with post hoc Bonferroni correction), respectively. ITT and ipGTT curves were compared using the repeated-measure ANOVA and by calculating the AUC. Linear correlations were checked using the Pearson's $r$ coefficients, which were compared using Fisher transformation. A multivariable linear regression analysis was carried out using 
IBM SPSS Statistics software (version 22.0, Chicago, IL, USA). Statistical significance was set at $p<0.05$.

\section{Results}

Development of obesity in p66Shc ${ }^{-/-}$and WT mice p66Shc $c^{-1-}$ mice were slightly leaner $(\sim 10 \%$ lower body weight, $n=15-30$ mice/time point) compared with WT mice from about 10-24 weeks of age (Fig. 1a). In the Lep ${ }^{W T / W T}$ strain, $p 66 \mathrm{Sh}^{-1-}$ mice had markedly reduced plasma leptin concentrations at both 18 and 30 weeks of age (ESM Fig. 1). When leptin-deficient mice (Lep ${ }^{o b / o b}$, from now on referred to as $o b / o b$ ) were analysed, starting from week 18 , $p 66 \mathrm{Sh}^{-1-} \mathrm{ob} / \mathrm{ob}$ mice gained less weight compared with $o b /$ $o b$ mice, and body weight was reduced by $20 \%$ at week 30 (Fig. 1a).

Metabolic features of lean and obese $p 66 \mathrm{Shc}^{--}$and WT mice We analysed ectopic fat accumulation in metabolically active organs, such as the liver and skeletal muscle. $\mathrm{Ob} / \mathrm{ob}$ mice showed severe hepatic triacylglycerol overload ( $>$ tenfold increase), which was nonsignificantly improved by p66Shc deletion at $18(p=0.35)$ and $30(p=0.06)$ weeks (Fig. 1b, c). Further evidence was provided by Oil Red O staining, which indicated that $p 66 \mathrm{Sh}^{-1-}$ mice are not protected from hepatic lipid accumulation induced by overfeeding (Fig. 1f, g). An increase in triacylglycerol content
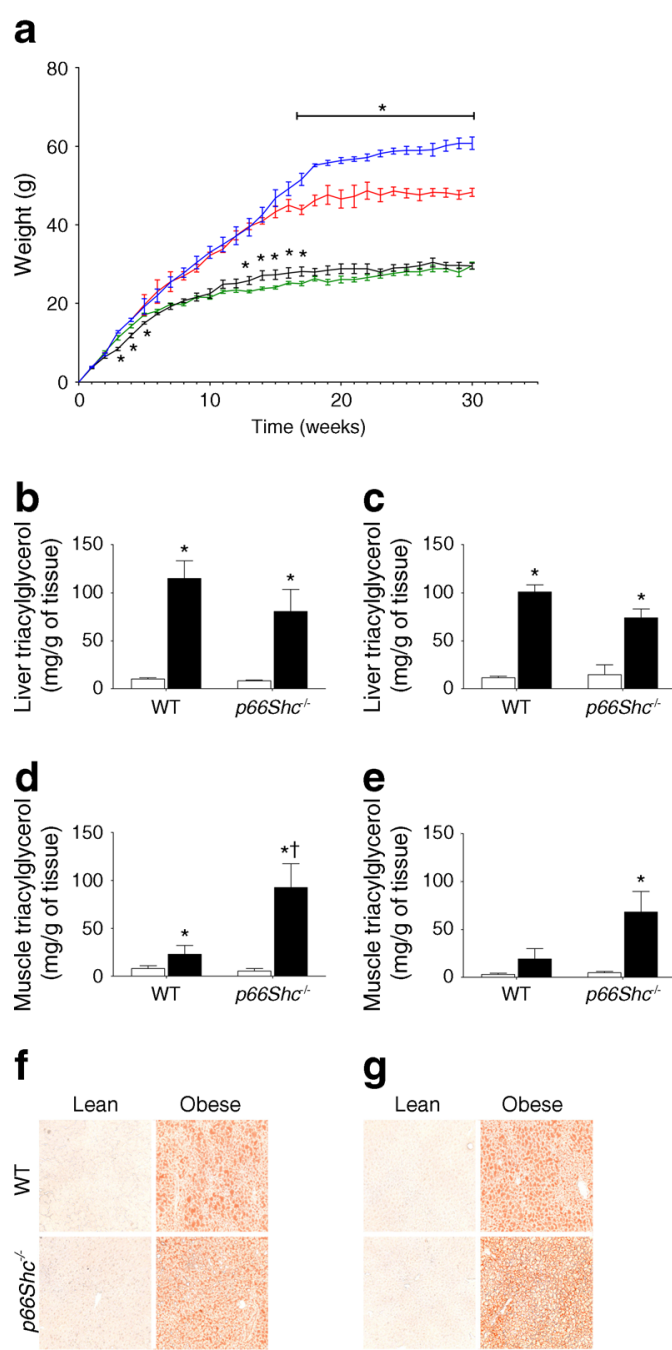

Fig. 1 (a) Body weight change over time in WT (lean, black; $o b / o b$, blue) and $p 66 \mathrm{Sh}^{-/-}$(lean, green; $o b / o b$, red) mice. ${ }^{*} p<0.05$ comparing $o b / o b$ with lean mice (either WT or $p 66 \mathrm{Sh}^{-/-}$). (b-e) Ectopic fat accumulation in the liver $(\mathbf{b}, \mathbf{c})$ and muscle $(\mathbf{d}, \mathbf{e})$, measured as tissue triacylglycerol content at $18(\mathbf{b}, \mathbf{d})$ and $30(\mathbf{c}, \mathbf{e})$ weeks of age. ${ }^{*} p<0.05$ for $o b /$ $o b$ vs lean. ${ }^{\dagger} p<0.05$ for $p 66 S h c^{-/-}$vs WT. (f-g) Representative Oil Red O staining of liver sections obtained from lean and $o b / o b \mathrm{WT}$ and $p 66 \mathrm{Sh} \mathrm{c}^{-1-}$ mice at 18 (f) and $30(\mathbf{g})$ weeks of age. Each picture $(\mathbf{f}, \mathbf{g})$ shows an area of
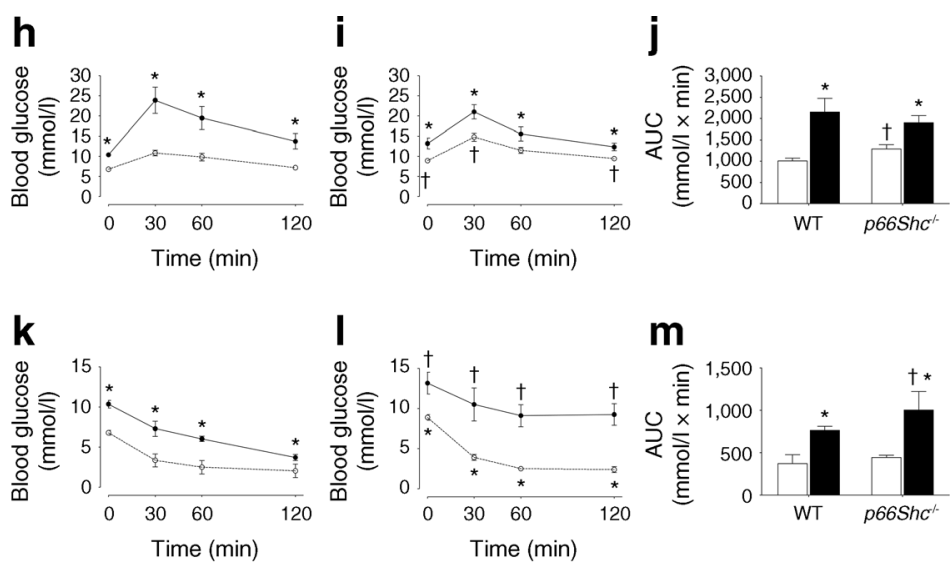

$\mathbf{m}$
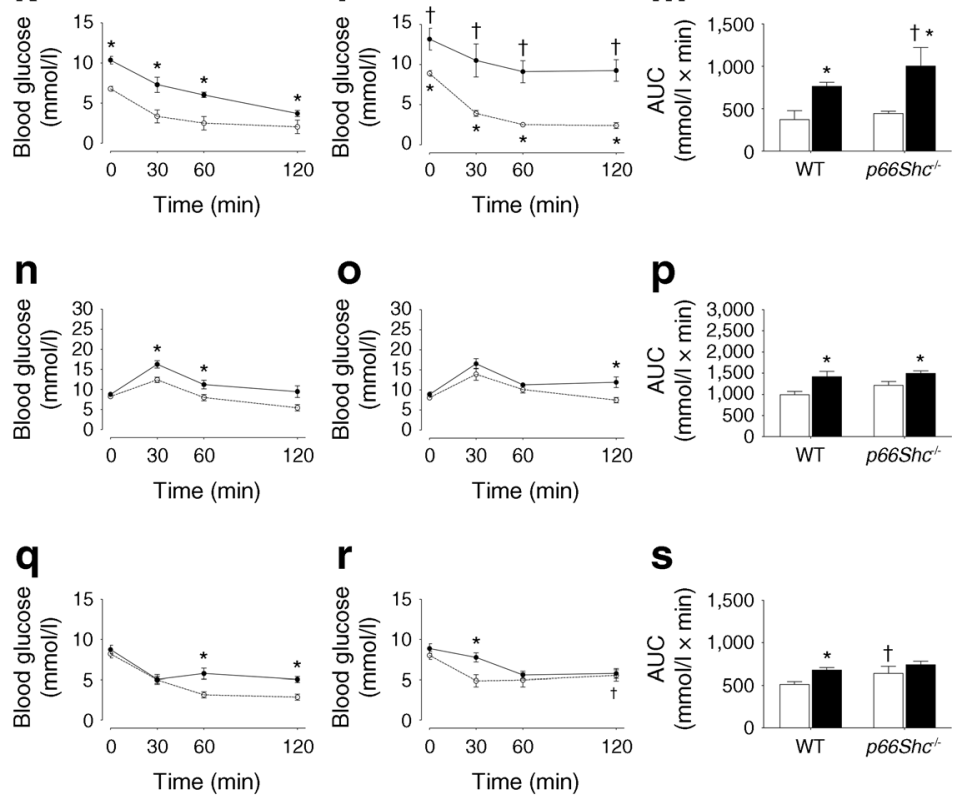

$670 \times 670 \mu \mathrm{m}$. (h-s) Metabolic function tests performed at $18(\mathbf{h}-\mathbf{m})$ and $30(\mathbf{n}-\mathbf{s})$ weeks of age. $(\mathbf{h}-\mathbf{j}, \mathbf{n}-\mathbf{p})$ ipGTTs; $(\mathbf{k}-\mathbf{m}, \mathbf{q}-\mathbf{s})$ ITTs. $(\mathbf{h}, \mathbf{k}, \mathbf{n}, \mathbf{q})$ Data from WT mice; $(\mathbf{i}, \mathbf{l}, \mathbf{o}, \mathbf{r})$ data from $p 66 S h c^{-1-}$ mice; $(\mathbf{j}, \mathbf{m}, \mathbf{p}, \mathbf{s})$ AUC data for all groups. Following two-way ANOVA, ${ }^{*} p<0.05$ for $o b / o b$ vs lean, ${ }^{\dagger} p<0.05$ for $p 66 S^{-1-}$ vs WT. White bars and dashed lines with white circles indicate lean mice; black bars and continuous lines with black circles indicate $o b / o b$ mice 
( $\sim$ threefold) was also found, as expected, in the skeletal muscles of $o b / o b$ compared with WT mice; this was significantly worsened by $p 66 \mathrm{Sh} c$ deletion at both 18 and 30 weeks (Fig. 1d, e). As determined by ex vivo insulin-induced glucose uptake, the $p 66 S h c^{-l-}$ skeletal muscle was insulin resistant compared with WT, especially at 30 weeks of age (ESM Fig. 2).

Fasting blood glucose levels were higher in $o b / o b$ compared with lean mice, and were significantly higher in lean p66Shc ${ }^{-1-}$ compared with WT mice at both 18 and 30 weeks and in $o b / o b p 66 \mathrm{Shc}^{-1-}$ compared with WT mice at 18 weeks (ESM Fig. 3). At both 18 and 30 weeks, ob/ob mice showed glucose intolerance compared with WT mice in ipGTTs (Fig. 1h-j, n-p). Glucose tolerance of 18-week-old lean p66Shc ${ }^{-1-}$ mice was worse than that of WT mice of the same age, and $p 66$ Shc deletion did not prevent the effects of obesity, as $p 66 \mathrm{Shc}^{-1-}$ ob/ob mice were less glucose tolerant compared with lean mice (Fig. 1h, j). At the same time, $p 66 \mathrm{Shc}^{-/-} \mathrm{ob} / \mathrm{ob}$ mice showed insulin resistance in ITTs compared with lean mice (Fig. 1k-m, q-s), especially at 18 weeks, when they were more insulin resistant than WT obese mice (Fig. 1k-m). No significant differences were detected between male and female mice (not shown).

p66Shc $c^{-1-}$ mice were only mildly and transiently protected from hyperinsulinaemia induced by obesity, and showed hyperglucagonaemia by week 18 compared with WT mice. The insulin:glucagon ratio, which was increased by tenfold in $o b / o b$ vs WT mice, was reduced in $p 66 \mathrm{Sh}^{-/-} \mathrm{ob} / \mathrm{ob}$ mice vs $o b / o b(p=0.005)$ at 18 , but not at 30 weeks of age. HOMA-IR and QUICKI values showed that lean $p 66 S h c^{-1-}$ mice were already insulin resistant compared with controls and were not protected from obesity-induced worsening in insulin resistance (ESM Fig. 1). Lean $p 66 \mathrm{Sh}^{-1-}$ mice showed reduced resistin concentrations at 18 and 30 weeks compared with WT mice, but this difference was lost in obese mice. Circulating plasminogen activator inhibitor-I (PAI-I) concentrations were increased by obesity and tended to be higher in p66Shc $c^{-1-}$ mice (ESM Fig. 1). Metabolic signalling pathways were investigated using a multiplex phospho-protein array in extracts from liver and muscle tissues taken under fasting conditions from lean and $o b / o b$ mice at 18 and 30 weeks. These showed no substantial effects of $p 665 h c$ deletion on Akt, ERK, mTor or S6K activation (ESM Fig. 4). Taken together, these data indicate that, despite being significantly leaner, overfed leptin-deficient $p 66 S^{-1-} c^{-1}$ are not protected from insulin resistance and glucose intolerance, and show ectopic fat accumulation in the liver and muscles. To confirm these findings, we used the HFD model. During a 12 week HFD, $p 66 \mathrm{Shc}^{-1-}$ mice gained significantly less weight than WT mice. At the end of this period, HFD had induced glucose intolerance and insulin resistance in both WT and $p 66 \mathrm{Shc}^{-1-}$ mice, with no difference between the two strains (ESM Fig. 5).

Adipose tissue remodelling in obese $\mathrm{p} 66 \mathrm{Shc}^{-/-}$and WT mice The adipocyte cross-sectional area (CSA) is proportional to the degree of insulin resistance. In SAT and VAT, the average CSA was markedly increased in $o b / o b$ mice and was overall unaffected by $p 665 h c$ deletion at 18 and 30 weeks (Fig. 2a, d). When the distributions of adipocyte CSA were analysed, lean and obese $p 66 \mathrm{Shc}^{-/-}$ mice displayed more small adipocytes in SAT and VAT compared with WT mice at 18 weeks of age, but this difference was lost at 30 weeks, when $p 66 \mathrm{Shc}^{-1-} \mathrm{ob} / \mathrm{ob}$
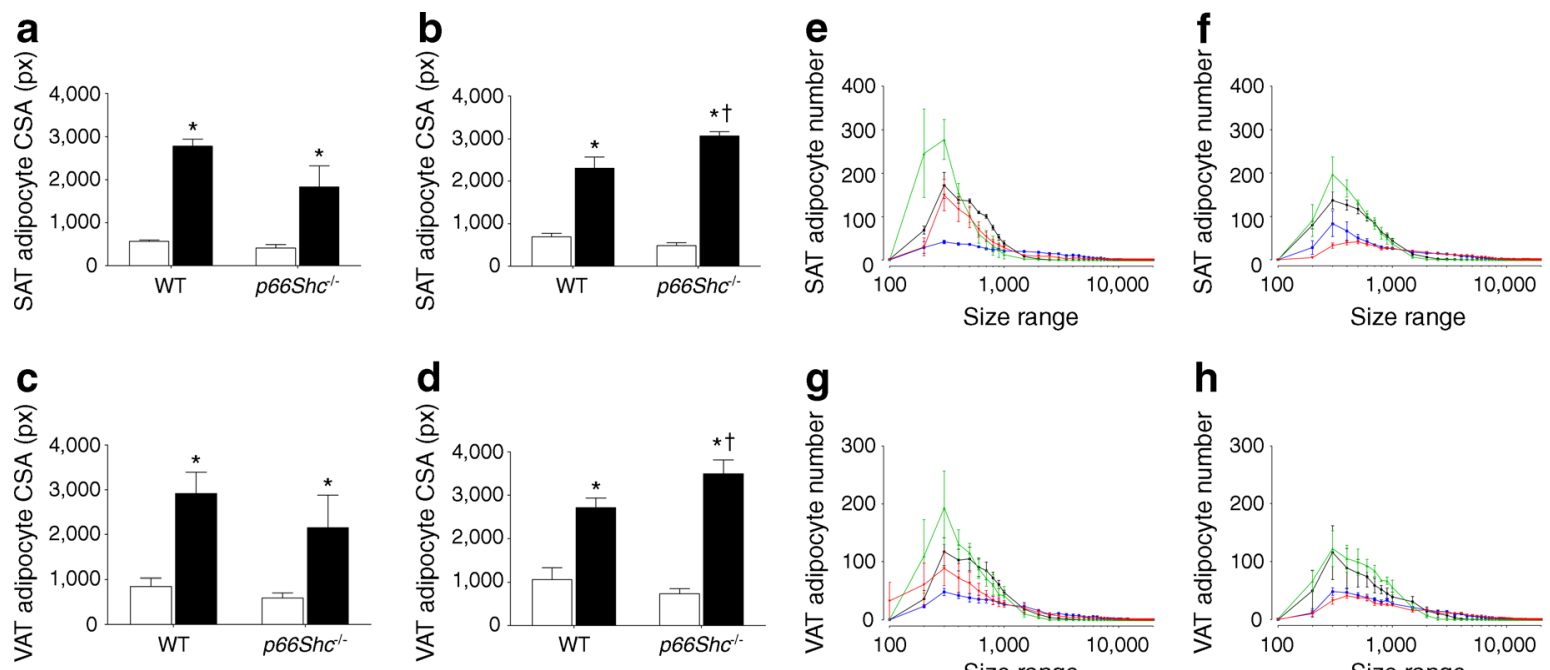

Fig. 2 (a-d) Average adipocyte CSA in SAT (a, b) and VAT (c, d) at 18 $(\mathbf{a}, \mathbf{c})$ and $30(\mathbf{b}, \mathbf{d})$ weeks of age. White bars, lean mice; black bars, $o b / o b$ mice. ${ }^{*} p<0.05$ for $o b / o b$ vs lean. Following two-way ANOVA, ${ }^{\dagger} p<0.05$ for 30 vs 18 weeks of age. (e-h) Distributions of adipocytes according to
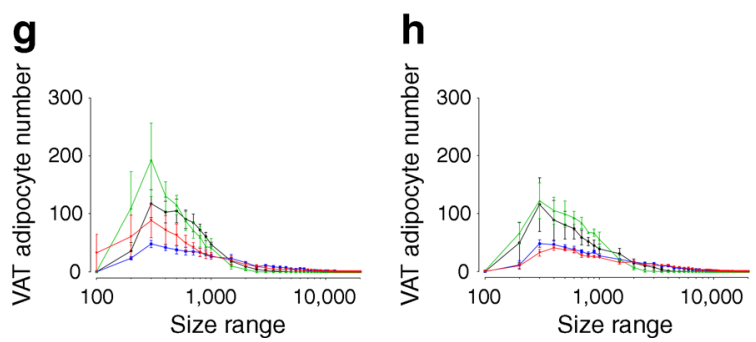

CSA class (on a log scale) in SAT $(\mathbf{e}, \mathbf{f})$ and VAT $(\mathbf{g}, \mathbf{h})$ at $18(\mathbf{e}, \mathbf{g})$ and 30 (f, h) weeks of age in WT (lean black; ob/ob, blue) and p66Shc ${ }^{-/-}$(lean, green; $o b / o b$, red). px, pixels 
mice showed the most right-skewed distribution of adipocyte CSA in both SAT and VAT (Fig. 2e, h).

Adipocyte apoptosis is thought to drive adipose tissue inflammation. The frequency of $\mathrm{TUNEL}^{+}$apoptotic nuclei in SAT and VAT sections was increased in $o b / o b$ compared with WT mice, and this was blunted by p66Shc deletion only at 18 weeks (Fig. 3a, c, e, g). At 30 weeks, lean but not obese p66Shc ${ }^{-1}$ mice had fewer apoptotic cells in VAT (Fig. 3b, d, $\mathrm{f}, \mathrm{h})$. TUNEL ${ }^{+}$nuclei:adipocyte ratios of $>1$ indicated the presence of vascular or interstitial cell apoptosis. As shown by $\mathrm{F} 4 / 80^{+}$staining of adipose-tissue-infiltrating macrophages, the number of crown-like structures (CLS) per adipocyte was significantly increased in SAT and VAT from $o b / o b$ mice. The increased CLS:adipocyte ratio in $p 66 \mathrm{Sh}^{-/-} \mathrm{ob} / \mathrm{ob}$ compared with $p 66 \mathrm{Sh}^{-1-}$ lean mice was blunted in VAT, especially at 30 weeks (Fig. 3i-p).

Collagen staining with picrosirius red showed a trend toward induction of fibrosis in VAT but not in SAT of $o b / o b$ compared with WT mice. Compared with WT $o b / o b$ mice, $p 66 \mathrm{Shc}^{-/-}$ob/ob mice had a higher SAT collagen content and no protection against VAT fibrosis at 18 and 30 weeks (Fig. 4a-h).
Adipose tissue haemoglobin content was measured as a surrogate of tissue perfusion. Obesity induced an increase in haemoglobin content, which was blunted by p66Shc deletion, especially in SAT at 18 weeks and in VAT at 30 weeks (Fig. 4i, 1).

Correlation between p66Shc expression and human adiposity In a parallel analysis, we analysed p66Shc gene and protein expression in VAT biopsies obtained from 77 patients undergoing abdominal surgery (Table 1). We report that the correlation between mRNA and protein expression was weak $\left(r^{2}=0.21\right)$, suggesting that post-transcriptional regulation accounts for changes in p66Shc protein levels. A mild positive correlation was found between BMI and p66Shc gene $(r=$ $0.22 ; p=132 ; n=46)$ and protein $(r=0.27 ; p=0.014 ; n=77)$ expression. Although non-obese participants (BMI $<30 \mathrm{~kg} /$ $\mathrm{m}^{2}$ ) had a lower p66Shc protein content in VAT (Fig. 5a, b), the association between BMI and p66Shc protein levels was present only in patients without the metabolic syndrome (Fig. 5c, d). $r$ values between VAT p66Shc protein and BMI were significantly different in patients with and without the metabolic syndrome $(Z=2.12, p=0.034$; Fig. 5c). Finally,
Fig. 3 (a-h) Apoptosis, determined as the number of $\mathrm{TUNEL}^{+}$nuclei per adipocyte, in $\operatorname{SAT}(\mathbf{a}, \mathbf{b}, \mathbf{e}, \mathbf{f})$ and $\operatorname{VAT}(\mathbf{c}, \mathbf{d}, \mathbf{g}$, h) at $18(\mathbf{a}, \mathbf{c}, \mathbf{e}, \mathbf{g})$ and $30(\mathbf{b}, \mathbf{d}, \mathbf{f}$, h) weeks of age. White bars, lean mice; black bars, ob/ob mice. (eh) Representative histological images. (i-p) Macrophage infiltration, determined as the number of $\mathrm{F} 4 / 80^{+} \mathrm{CLS}$ per adipocyte, in the SAT $(\mathbf{i}, \mathbf{j}, \mathbf{m}, \mathbf{n})$ and $\operatorname{VAT}(\mathbf{k}, \mathbf{l}, \mathbf{o}, \mathbf{p})$ at $18(\mathbf{i}, \mathbf{k}, \mathbf{m}$, o) weeks of age. $(\mathbf{m}-\mathbf{p})$ Representative histological images. Following two-way ANOVA, ${ }^{*} p<0.05$ for $o b / o b$ vs lean; ${ }^{\dagger} p<0.05$ for $p 66 \mathrm{Sh}^{-/-}$vs WT. Scale bars in (h) and (p), $100 \mu \mathrm{m}$ for parts $(\mathbf{e}-\mathbf{h})$ and $(\mathbf{m}-\mathbf{p})$
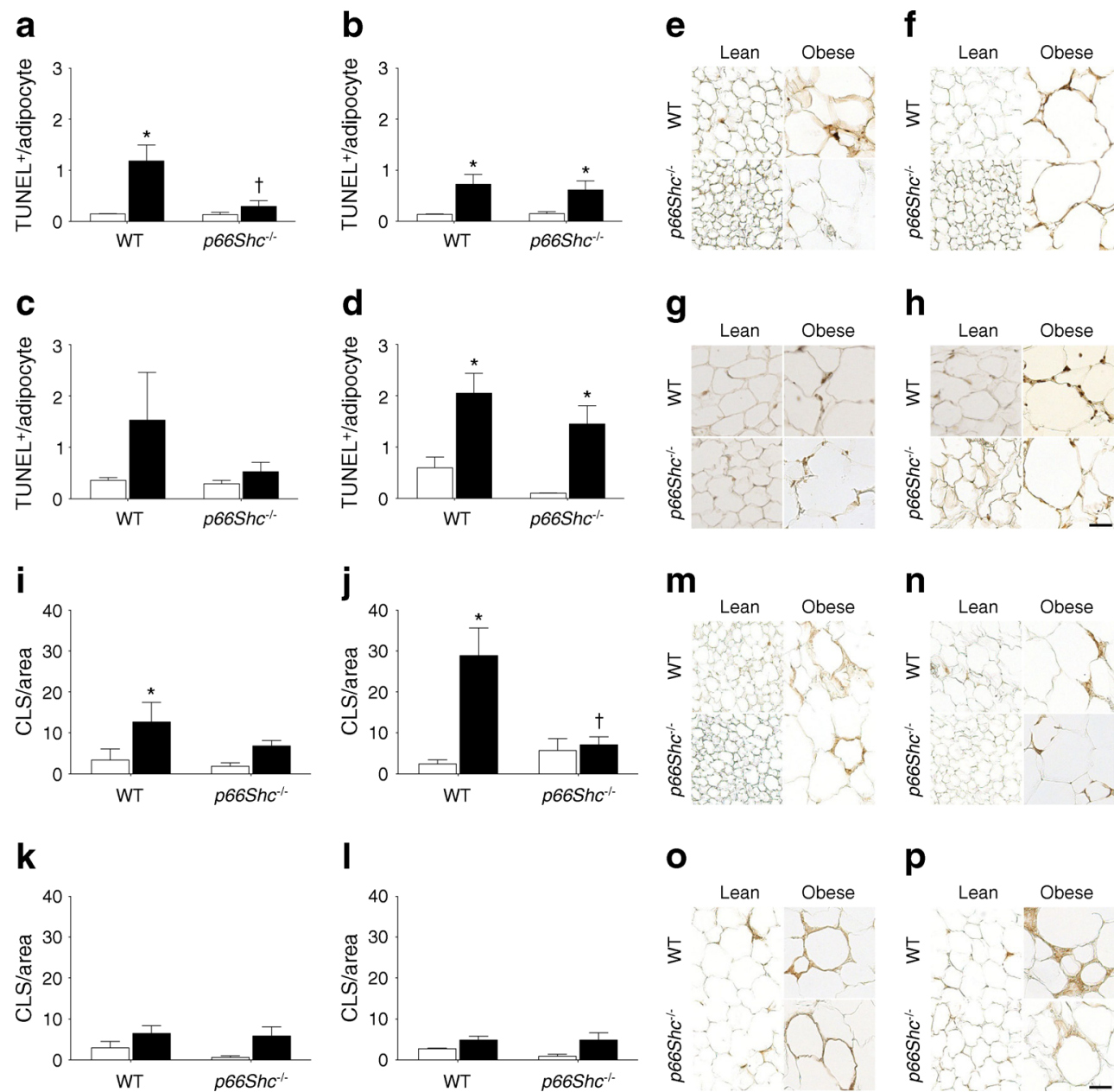

I

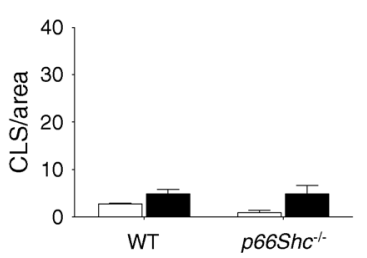

o

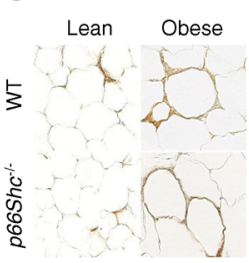

h

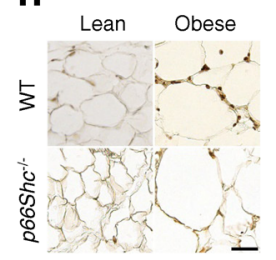

n

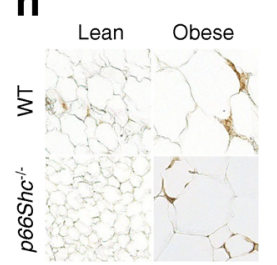

p

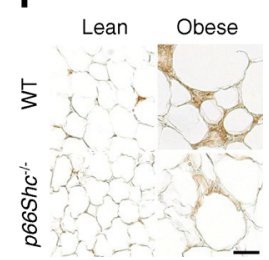


Fig. 4 (a-h) Fibrosis, determined as the percentage area of interstitial picrosirius red staining, in SAT (a, b, e, f) and $\operatorname{VAT}(\mathbf{c}, \mathbf{d}, \mathbf{g}, \mathbf{h})$ at $18(\mathbf{a}, \mathbf{c}, \mathbf{e}, \mathbf{g})$ and $30(\mathbf{b}, \mathbf{d}, \mathbf{f}, \mathbf{h})$ weeks of age. White bars, lean mice; black bars, $o b / o b$ mice. (e-h) Representative histological images. (i-l) SAT (i, j) and VAT $(\mathbf{k}, \mathbf{l})$ perfusion, determined as tissue haemoglobin content at $18(\mathbf{i}, \mathbf{k})$ and $30(\mathbf{j}, \mathbf{l})$ weeks of age. Following two-way ANOVA, ${ }^{*} p<0.05$ for $o b / o b$ vs lean; ${ }^{\dagger} p<0.05$ for $p 66 \mathrm{Sh}^{-/-}$vs $\mathrm{WT} ;{ }^{\ddagger} p<0.05$ for 30 vs 18 weeks of age. Scale bar in (h), $100 \mu \mathrm{m}$ for parts $(\mathbf{e}-\mathbf{h})$
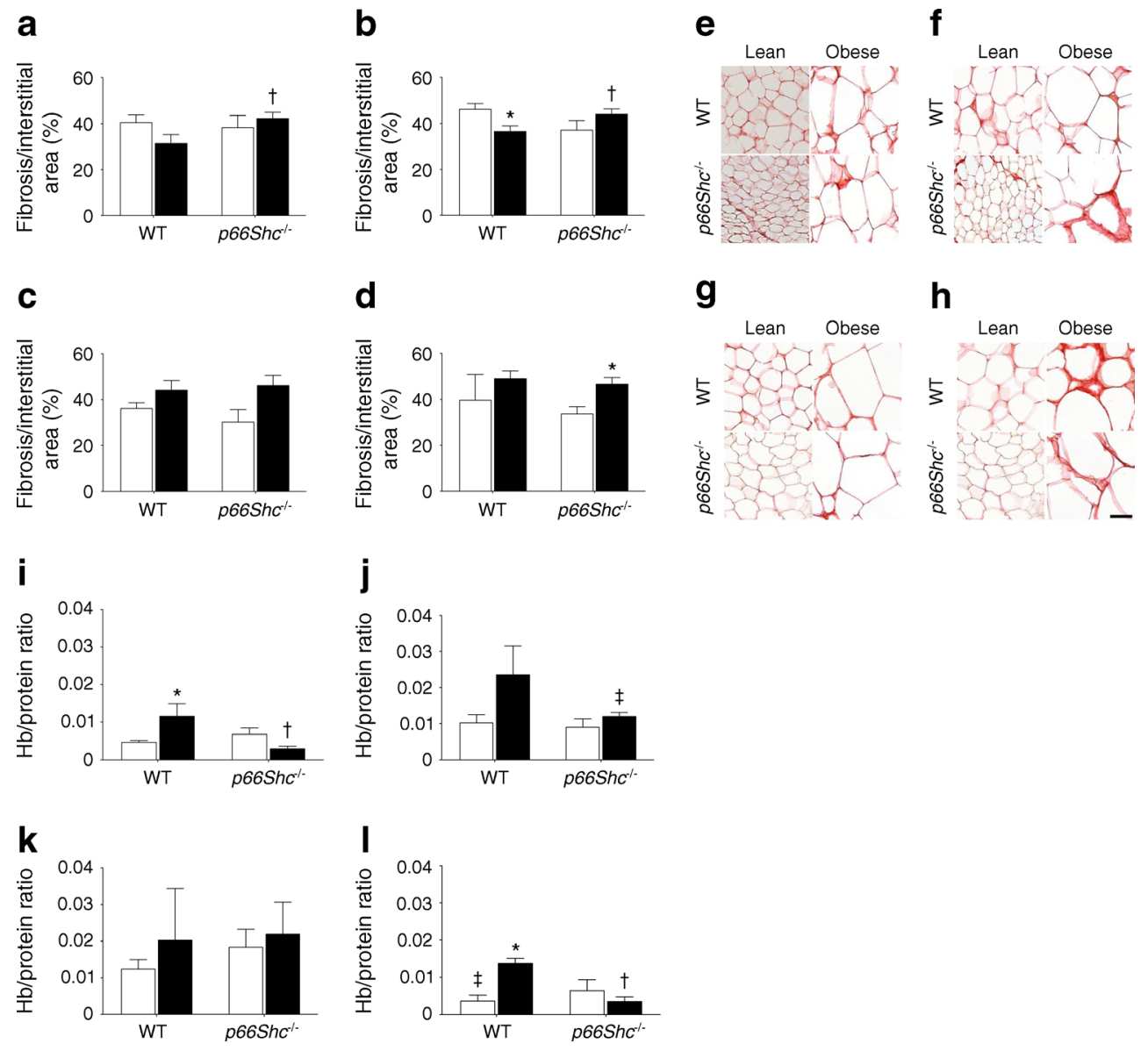

patients with low (below median) p66Shc protein content in VAT were less likely to be obese, but tended to be diabetic, dyslipidaemic and hypertensive (Fig. 5e). A multivariable analysis indicated that BMI was the only significant determinant of VAT p66Shc protein levels (Table 2). These data suggest that low p66Shc protein levels are associated with a leaner phenotype but do not confer protection against features of the metabolic syndrome.

Table 1 Clinical characteristics of study patients

\begin{tabular}{ll}
\hline Characteristic & Value \\
\hline Number & 77 \\
Age (range), years & $51.3 \pm 1.6(25-85)$ \\
Sex, \% male & 37.3 \\
BMI (range), kg/m² & $43.1 \pm 1.3(21.9-67.1)$ \\
Diabetes, \% & 35.1 \\
Dyslipidaemia, \% & 50.6 \\
Hypertension, \% & 64.9 \\
ATP-III metabolic syndrome, \% & 49.3 \\
Cardiovascular disease, \% & 33.7 \\
\hline
\end{tabular}

\section{Discussion}

This study shows that $p 66 \mathrm{Shc}^{-/-}$mice are protected from obesity and from some adverse features of adipose tissue remodelling, but accumulate ectopic fat in the liver and muscles and are not protected from insulin resistance and glucose intolerance induced by overfeeding. The dysmetabolic phenotype observed in $\mathrm{p} 66 \mathrm{Shc} \mathrm{c}^{-1-}$ mice despite the consistently lower body weight compared with WT mice in both the $o b / o b$ and HFD models is reminiscent of lipodystrophy. p66Shc generates $\mathrm{H}_{2} \mathrm{O}_{2}$, and ROS are required for adipocyte differentiation and lipid storage $[8,13]$. Therefore, $p 66 \mathrm{Shc}^{-1-}$ ob/ob mice gained less weight than their controls, probably because of a defect in adipocyte growth. As body mass was reduced but the average adipocyte area was not, adipocyte hyperplasia may be compromised by $p 66 \mathrm{Sh} c$ deletion. Previous data showed that p66Shc ${ }^{-1-}$ mice fed a standard diet [14] or a HFD had a normal food intake and a mildly elevated metabolic rate [8], whereas p66Shc deletion affected only the short-term metabolic effects of caloric restriction [15]. Therefore, lipids that are not stored in the adipose tissue are only partly oxidised and accumulate in ectopic sites, such as the liver and skeletal muscle. This impaired fatty acid trapping is a likely mechanism by which p66Shc ${ }^{-1-}$ mice become insulin resistant and glucose 
a

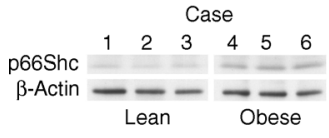

b
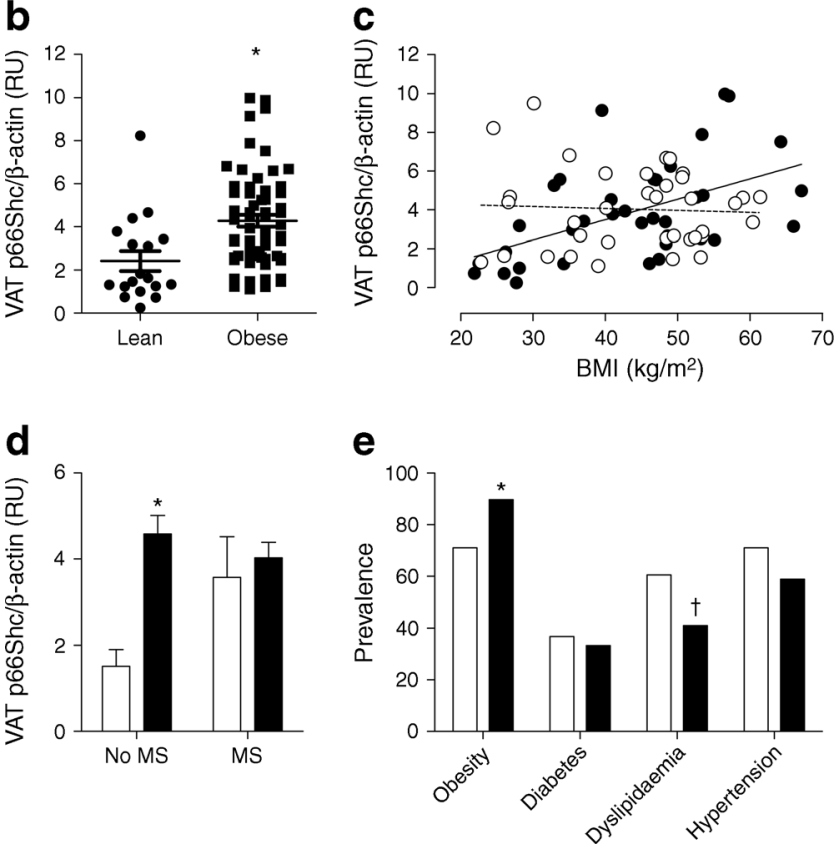

Fig. 5 p66Shc protein levels in VAT of patients $(n=77)$ undergoing elective surgery. $(\mathbf{a}, \mathbf{b})$ VAT p66Shc levels in lean $\left(\mathrm{BMI}<30 \mathrm{~kg} / \mathrm{m}^{2}\right)$ and obese (BMI $\left.\geq 30 \mathrm{~kg} / \mathrm{m}^{2}\right)$ participants $\left({ }^{*} p<0.05\right.$ ). (a) Representative western blot images of three lean and three obese patients. (c) Correlation between BMI and VAT p66Shc protein levels in patients with (white circles and dashed line; $r=0.02$ ) and without (black circles and solid line; $r=0.48$ ) the metabolic syndrome (MS). (d) VAT p66Shc protein levels in lean (BMI $<30 \mathrm{~kg} / \mathrm{m}^{2}$, white columns) and obese (BMI $\geq 30 \mathrm{~kg} / \mathrm{m}^{2}$, black columns) participants classified according to the presence/absence of the MS. ${ }^{*} p<0.05$ for obese vs lean. (e) Prevalence of obesity and other MS components in patients categorised as having low (below median, white columns) or high (above median, black columns) VAT p66Shc protein levels. ${ }^{*} p<0.05$ in a $\chi^{2}$ test; ${ }^{\dagger} p=0.08$. RU, relative units

intolerant. In fact, 18-week-old $p 66 \mathrm{Shc}^{-/-}$mice, which are about $10 \%$ leaner than WT mice, were already glucose intolerant even on a $L e p^{W T / W T}$ background. Previous studies

Table 2 Results of the multivariable analysis of clinical data

\begin{tabular}{lcc}
\hline Variable & Standardised $\beta$ coefficient & $p$ value \\
\hline Age & 0.056 & 0.700 \\
Sex, male & -0.123 & 0.351 \\
BMI & 0.276 & 0.044 \\
Diabetes & -0.200 & 0.177 \\
Dyslipidaemia & -0.217 & 0.112 \\
Hypertension & -0.149 & 0.286 \\
Cardiovascular disease & -0.018 & 0.906
\end{tabular}

VAT p66Shc protein content was the dependent variable, and covariates were entered as a single block reported better glucose tolerance and insulin sensitivity in p66Shc $c^{-1-}$ mice on the $\mathrm{Lep}^{\mathrm{ob} / \mathrm{ob}}$ background [11] or on a HFD [12] compared with WT mice. Possible reasons for the discrepancy with our results include differences in housing environment, the original background (C57Bl/6 vs $\mathrm{C} 57 \mathrm{Bl} /$ $6 \mathrm{~J})$ and the timing of observation. We would like to emphasise that this is the first study to analyse the parallel changes in body weight, glucose/insulin tolerance and adipose tissue histopathology during the development of obesity in $\mathrm{p} 66 \mathrm{Sh} \mathrm{c}^{-/-}$ mice, using both leptin-deficient and HFD models. Typical features of the expanding VAT in obesity include macrophage infiltration, apoptosis, fibrosis and angiogenesis [16, 17]. These changes favour fatty acid leakage and ectopic storage, leading to insulin resistance in the muscle and liver. Adipocyte area is proportional to adipose insulin resistance, is higher in VAT than in SAT and is increased in obese mice and humans [18]. Lean and obese $p 66 S h c^{-1-}$ mice had smaller adipocytes at age 18 weeks, but $p 66 S h c^{-/-}$ob/ob mice showed markedly enlarged adipocytes compared with $o b / o b$ mice at age 30 weeks. Although p66Shc regulates mitochondrial apoptosis and $p 66 S h c$ deletion or knockdown protects from apoptosis [19], we only found a partial and transient reduction of adipocyte apoptosis in $\mathrm{p} 66 \mathrm{Sh} \mathrm{c}^{-/-}$mice, possibly because death of adipocytes may occur passively as they become severely enlarged during weight gain and excess energy storage $[18,20]$. Therefore, worsening of glucose tolerance, insulin sensitivity and ectopic fat accumulation in $\mathrm{p} 66 \mathrm{Shc}^{-/-} \mathrm{ob} / \mathrm{ob}$ mice correlated with adipocyte hypertrophy, despite a relative improvement in more specific inflammatory features, namely macrophage infiltration and angiogenesis.

In human adipose tissue samples, we found that p66Shc gene and protein expression were poorly correlated, as previously shown in studies using peripheral blood mononuclear cells [21]. In a cohort of 77 patients undergoing elective surgery, we found a direct correlation between BMI and VAT p66Shc protein levels, especially in the absence of the metabolic syndrome. Despite VAT p66Shc protein levels being lower in non-obese compared with obese patients, having a low p66Shc content did not protect patients from other features of the metabolic syndrome, which tended to be more prevalent in patients with low p66Shc levels than in those with higher p66Shc levels. This finding strikingly parallels those obtained with $p 665 h c^{-1-}$ mice, which became less obese than WT mice but still accumulated ectopic fat and became glucose intolerant. It should be noted that we used the $S h c P$ knockout strain originally described to be long-lived and protected from oxidative stress related diseases [3]. This strain seems to have a different phenotype compared with $S h c L$ knockout mice with respect to fat accumulation [12]. This difference has been attributed to upregulation of p46Shc in the adipose tissue of p66Shc $c^{-1-}$ mice [12]. However, we were unable to confirm $\mathrm{p} 46 \mathrm{Shc}$ upregulation in the adipose tissue of $\mathrm{p} 66 \mathrm{Shc}^{-/-}$mice (ESM Fig. 6), and no role for p46Shc in obesity has been 
demonstrated so far. In addition, p66Shc may have divergent, and possibly antagonistic, effects in the different organs and tissues involved in the metabolic response to overfeeding, an issue that could be addressed only with tissue-specific $p 66 \mathrm{Sh} c$ knockout. For instance, obese $p 66 \mathrm{Sh}^{-/-}$mice become glucose intolerant despite $p 66 \mathrm{Sh}^{-1-}$ beta cells being resistant to lipotoxicity-induced apoptosis [22]. Therefore, further studies are still needed to dissect the role of p66Shc in whole body metabolism.

In summary, our findings in mice and humans collectively indicate that reduced p66Shc expression protects from obesity, possibly by inhibiting ROS-dependent adipogenesis, but does not protect from insulin resistance and glucose intolerance.

Acknowledgements We thank K. A. Dyar, Helmholtz Center, Munich, and L. E. Wright, Department of Biomedical Sciences, University of Padua, for technical assistance.

Funding This study was funded by an Italian Ministry of Health grant (no. GR-2010-2301676) to GPF.

Duality of interest statement The authors declare that there is no duality of interest associated with this manuscript.

Contribution statement All author provided substantial contributions to conception and design, acquisition of data or analysis and interpretation of data; drafting the article or revising it critically. SC, MA, AD, VS and MG performed experiments in animals, and analysed and interpreted data. LM, NP, EP, MM and BB designed, performed, analysed and interpreted in vitro experiments. MT, MF, LP and DN collected, analysed and interpreted human data. AA and GPF conceived the study, collected and interpreted data, and provided support. SC, MA and GPF wrote the manuscript. All authors reviewed and approved the final draft of the article. GPF is the guarantor of this work.

\section{References}

1. Migliaccio E, Giorgio M, Pelicci PG (2006) Apoptosis and aging: role of p66Shc redox protein. Antioxid Redox Signal 8:600-608

2. Giorgio M, Migliaccio E, Orsini F et al (2005) Electron transfer between cytochrome $\mathrm{c}$ and p66Shc generates reactive oxygen species that trigger mitochondrial apoptosis. Cell 122:221-233

3. Migliaccio E, Giorgio M, Mele S et al (1999) The p66 shc adaptor protein controls oxidative stress response and life span in mammals. Nature 402:309-313

4. Albiero M, Poncina N, Tjwa M et al (2014) Diabetes causes bone marrow autonomic neuropathy and impairs stem cell mobilization via dysregulated p66Shc and Sirt1. Diabetes 63:1353-1365
5. Fadini GP, Albiero M, Menegazzo L et al (2010) The redox enzyme p66Shc contributes to diabetes and ischemia-induced delay in cutaneous wound healing. Diabetes 59:2306-2314

6. Rota M, LeCapitaine N, Hosoda T et al (2006) Diabetes promotes cardiac stem cell aging and heart failure, which are prevented by deletion of the p66 shc gene. Circ Res 99:42-52

7. Menini S, Amadio L, Oddi G et al (2006) Deletion of p66Shc longevity gene protects against experimental diabetic glomerulopathy by preventing diabetes-induced oxidative stress. Diabetes 55 : $1642-1650$

8. Berniakovich I, Trinei M, Stendardo M et al (2008) p66Shcgenerated oxidative signal promotes fat accumulation. J Biol Chem 283:34283-34293

9. Giorgio M, Berry A, Berniakovich I et al (2012) The p66Shc knocked out mice are short lived under natural condition. Aging Cell 11:162-168

10. Neel JV (1962) Diabetes mellitus: a 'thrifty' genotype rendered detrimental by 'progress'? Am J Hum Genet 14:353-362

11. Ranieri SC, Fusco S, Panieri E et al (2010) Mammalian life-span determinant p66 shcA mediates obesity-induced insulin resistance. Proc Natl Acad Sci U S A 107:13420-13425

12. Tomilov AA, Ramsey JJ, Hagopian K et al (2011) The Shc locus regulates insulin signaling and adiposity in mammals. Aging Cell 10:55-65

13. Titta L, Trinei M, Stendardo $M$ et al (2010) Blood orange juice inhibits fat accumulation in mice. Int J Obes (Lond) 34:578-588

14. Stern JH, Kim K, Ramsey JJ (2012) The influence of shc proteins and aging on whole body energy expenditure and substrate utilization in mice. PLoS One 7:e48790

15. Stern JH, Kim K, Ramsey JJ (2012) The influence of acute, late-life calorie restriction on whole body energy metabolism in p66Shc(-/-) mice. Mech Ageing Dev 133:414-420

16. Sun K, Kusminski CM, Scherer PE (2011) Adipose tissue remodeling and obesity. J Clin Invest 121:2094-2101

17. Spencer M, Unal R, Zhu B et al (2011) Adipose tissue extracellular matrix and vascular abnormalities in obesity and insulin resistance. J Clin Endocrinol Metab 96:E1990-E1998

18. Cinti S, Mitchell G, Barbatelli G et al (2005) Adipocyte death defines macrophage localization and function in adipose tissue of obese mice and humans. J Lipid Res 46:2347-2355

19. Trinei M, Migliaccio E, Bernardi P et al (2013) p66Shc, mitochondria, and the generation of reactive oxygen species. Methods Enzymol 528:99-110

20. Jo J, Shreif Z, Periwal V (2012) Quantitative dynamics of adipose cells. Adipocytes 1:80-88

21. Pagnin E, Fadini G, de Toni R et al (2005) Diabetes induces p66shc gene expression in human peripheral blood mononuclear cells: relationship to oxidative stress. J Clin Endocrinol Metab 90: $1130-1136$

22. Natalicchio A, Tortosa F, Labarbuta R et al (2015) The p66(Shc) redox adaptor protein is induced by saturated fatty acids and mediates lipotoxicity-induced apoptosis in pancreatic beta cells. Diabetologia 58:1260-1271 